\title{
Ganar o perder en los torneos de Grand Slam en el tenis en silla de ruedas
}

\author{
Alejandro Sánchez, Antonio Ortega y David Sanz
}

\author{
Universidad de Murcia, Murcia, España
}

RESUMEN

En 2016 fue la primera vez que el torneo de Wimbledon incluyó la modalidad individual de Tenis en Silla de Ruedas (TSR) dentro del programa de su competición, por lo que a día de hoy este deporte tiene presencia en los tres tipos de superficie principales (pista dura, tierra batida, y hierba). Las estadísticas de competición pueden ayudar a entender las diferencias en el juego en función de la superficie. Por tanto, el objetivo de este trabajo será observar las posibles diferencias en el servicio entre los ganadores y perdedores en la élite del TSR tanto en masculino como en femenino en diferentes superficies. Se analizaron un total de 42 partidos compuestos por 101 sets de tres de los Grand Slam de 2016: Australia Open (AO), Roland Garros (RG) y Wimbledon (W). Los resultados mostraron que entre ganadores y perdedores el rendimiento varía en función de la superficie en la que se juega. Las conclusiones de este trabajo pueden ayudar a los entrenadores a adaptar sus entrenamientos en función de la superficie de la competición.

\author{
Palabras clave: Deporte \\ adaptado, rendimiento, \\ superficies, tenis. \\ Recibido: 29 Dic 2017 \\ Aceptado: 10 Mar 2018 \\ Autor correspondiente: \\ Alejandro Sánchez, \\ Universidad de Murcia, Murcia, \\ España. \\ Correo electrónico: \\ aspay@um.es
}

\section{INTRODUCCIÓN}

El tenis en silla de ruedas (TSR) se puede jugar en diferentes superficies (pista de cemento, moqueta, hierba y pista de tierra batida). Desde el año 2016, el TSR tiene presencia los 4 Grand Slams (GS) (Open de Australia, Roland Garros, US Open y Wimbledon). En los torneos mencionados anteriormente, existen diferencias por cuanto se refiere a la velocidad de la pelota tras el bote en el suelo, así como las características de los desplazamientos de los jugadores, y todo ello se puede resumir en lo que conocemos como el ritmo que impone la superficie de juego: 1 (ritmo pausado), 2 (ritmo medio pausado), 3 (ritmo medio), 4 (ritmo medio-acelerado), y 5 (ritmo acelerado). En este sentido, el estudio de las estadísticas de competición han ofrecido información importante a la hora de determinar las posibles diferencias en función de la superficie de juego (Sánchez-Pay, Palao, Torres-Luque, \& Sanz-Rivas, 2015) o establecer posibles indicadores de rendimiento entre ganadores y perdedores (Sánchez-Pay, Torres-Luque, Cabello Manrique, Sanz-Rivas, \& Palao, 2015).
Algunas investigaciones muestran diferencias significativas entre los cuatro torneos de GS, observando diferencias en la velocidad de las superficies de juego. El torneo de Roland Garros se juega en una superficie de tierra batida (superficie lenta), Wimbledon se juega en una superficie de hierba más rápida y el US Open y Australia se juega en una superficie dura de velocidad media, lo que hace que varíen aspectos de eficacia y efectividad técnica (Cross \& Pollard, 2009).

La primera vez que se ha disputado un torneo de TSR en la modalidad de individuales sobre superficie de hierba ha sido en el torneo de Wimbledon disputado en el año 2016, no habiéndose encontrado ningún estudio que compare la influencia de dicha superficie sobre las estadísticas de la competición. Por lo tanto, el objetivo de este trabajo será observar las posibles diferencias del rendimiento de los jugadores de élite del TSR en diferentes superficies de juego y centrándonos en uno de los golpes que puede marcar la diferencia como es el servicio, tanto el primero como el segundo, para comprobar su eficacia como indicador de rendimiento en el TSR de individuales y sobre diferentes superficies. 


\section{METODOLOGÍA}

La muestra estuvo formada por 48 jugadores de TSR (24 masculino y 24 femenino). Se analizaron el $100 \%$ de los partidos jugados en los torneos de Australia Open, Roland Garros, y Wimbledon de la temporada 2016 (tabla 1). Es importante señalar que en la modalidad de TSR los Grand Slams son sólo disputados por ocho jugadores, los mejores clasificados en el ranking ITF (ITF, 2018). El estudio se realizó de acuerdo con la declaración de Helsinki, y todos los procedimientos fueron aprobados por la Comisión de Bioética e Investigación de la Universidad de Murcia.

\begin{tabular}{cccc}
\hline & $\begin{array}{c}\text { Australian } \\
\text { Open }\end{array}$ & Roland Garros & Wimbledon \\
\cline { 2 - 4 } Masculino & 7 & 7 & 7 \\
Femenino & 7 & 7 & 7 \\
\hline
\end{tabular}

Tabla 1. Número de partidos de TSR analizados por torneos y género.

La muestra fue dividida en subgrupos para analizarlos: a) torneo: Australia Open (AO), Roland Garros (RG) y Wimbledon $(W) . y$ b) resultado: ganador del set o perdedor del set.

Todos los datos estadísticos de competición fueron extraídos de la información publicada en las Webs Oficiales de cada uno de los torneos (www.usopen.org,www.rolandgarros.com y www.wimbledon.com), al igual que estudios previos sobre el análisis de las estadísticas de competición en tenis (Cross \& Pollard, 2009; Knight \& O'Donoghue, 2012). Se realizó el test de Wilcoxon para analizar las diferencias entre los ganadores y los perdedores. La unidad de análisis fue el set y la significancia se estableció en $\mathrm{p}<.05$.

\section{RESULTADOS}

En las siguientes figuras se muestran las diferencias en las variables objeto de estudio (\% de primer servicio, \% de puntos ganados con primer y segundo servicio y $\%$ de puntos de break ganados) tanto en masculino como en femenino así como aquellas en las que existen diferencias estadísticamente significativas $(p<.05)$.

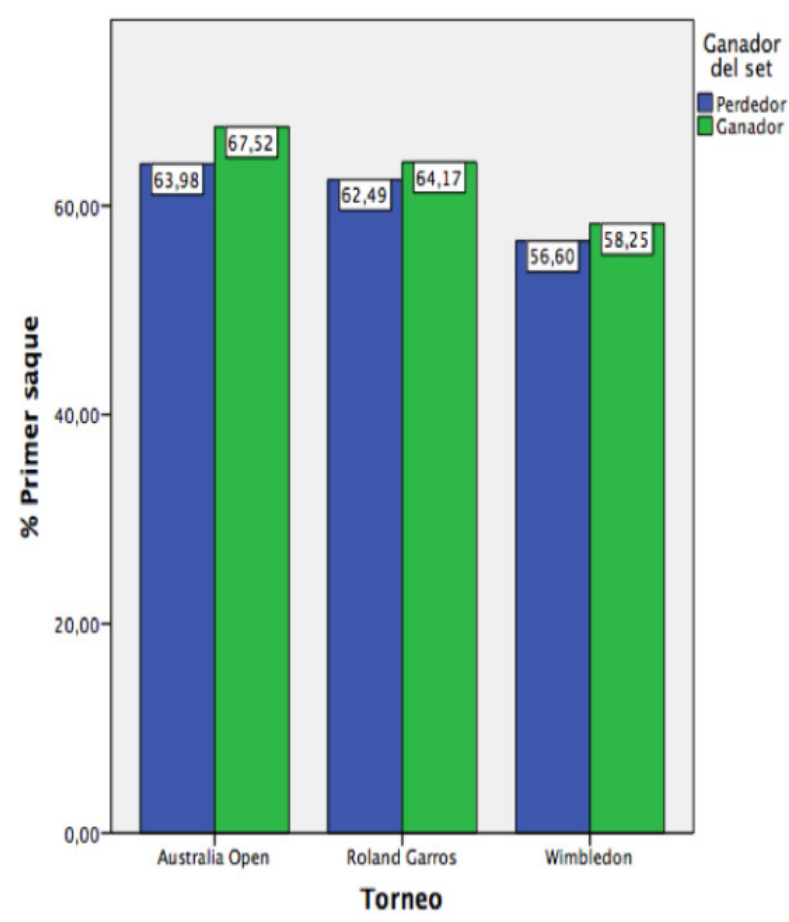

Figura 1. Descripción del \% primer saque en el TSR masculino.

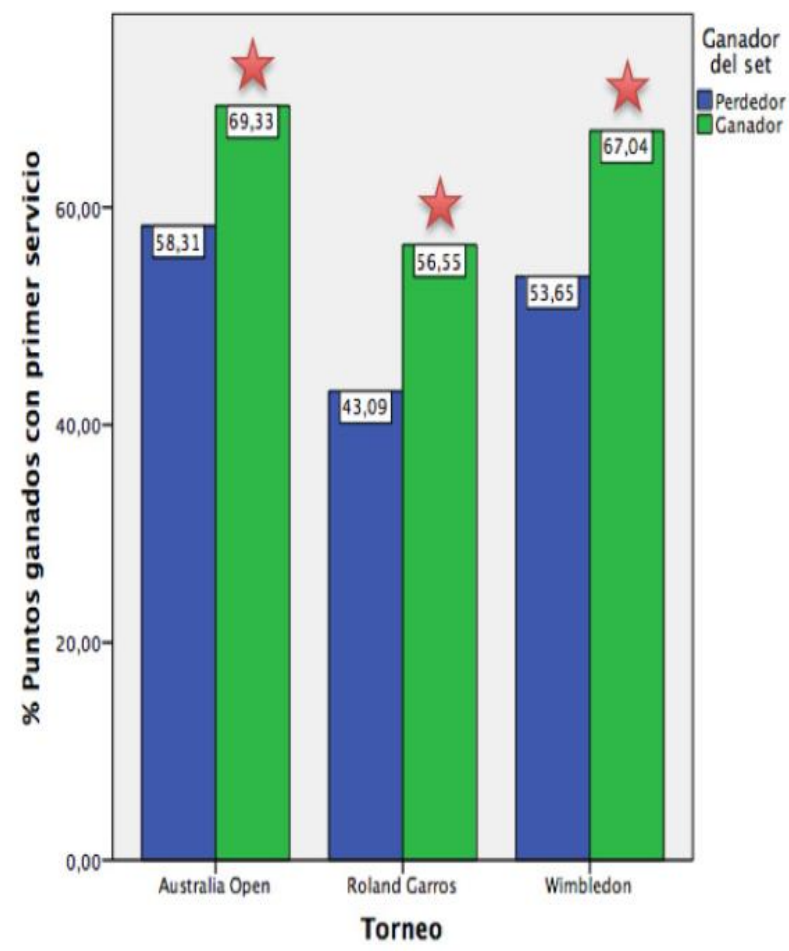

Figura 2. Descripción del \% puntos ganados con primer servicio en el TSR masculino. 


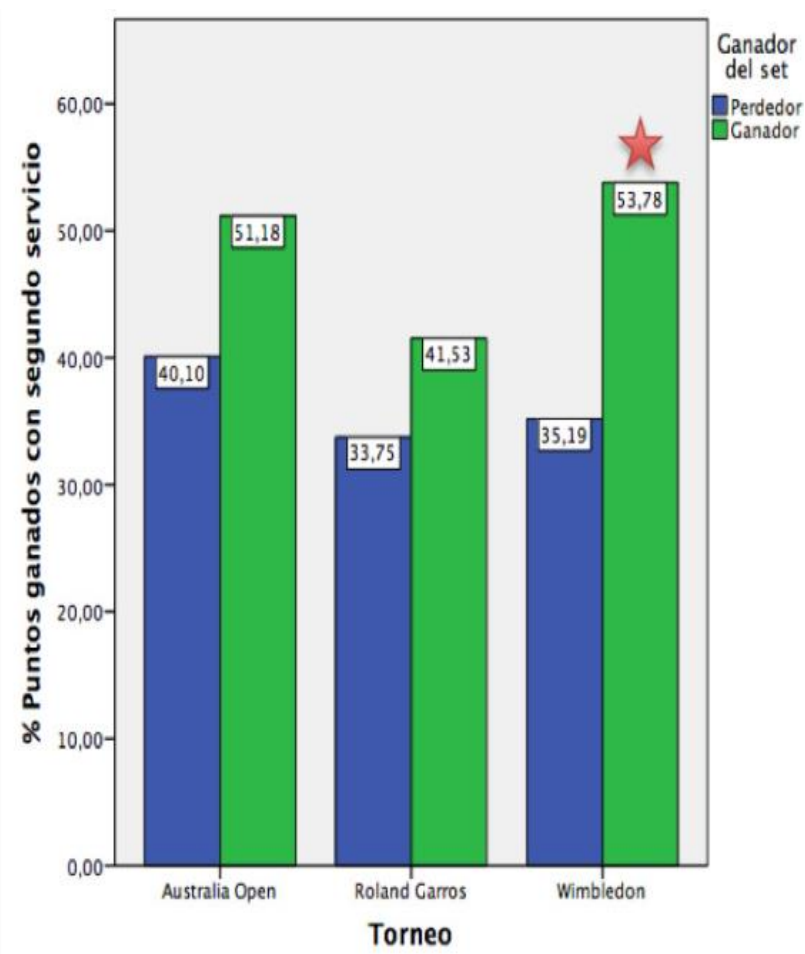

Figura 3. Descripción del \% puntos ganados con el segunde servicio en el TSR masculino.

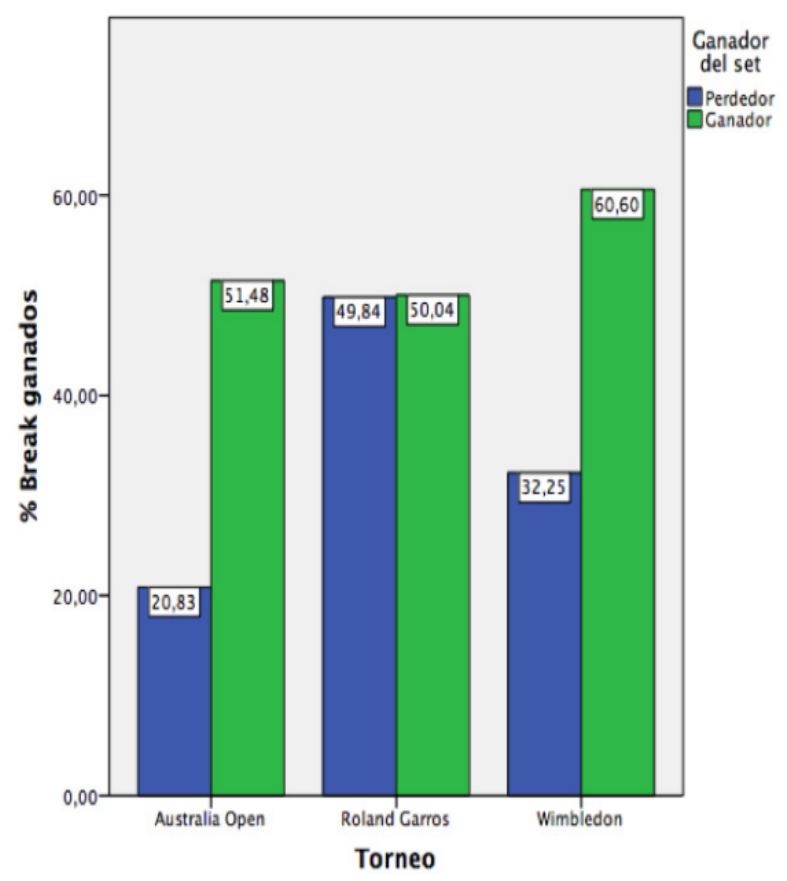

Figura 4. Descripción del \% rotura ganados en el TSR masculino.

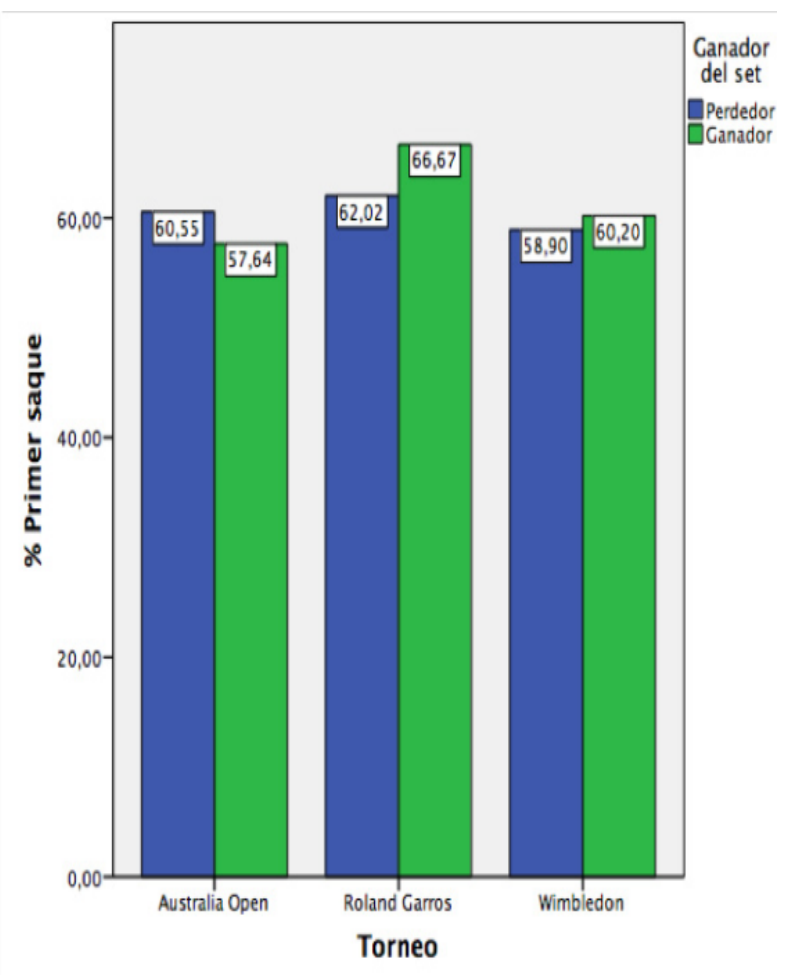

Figura 5. Descripción del \% primer saque del TSR femenino.

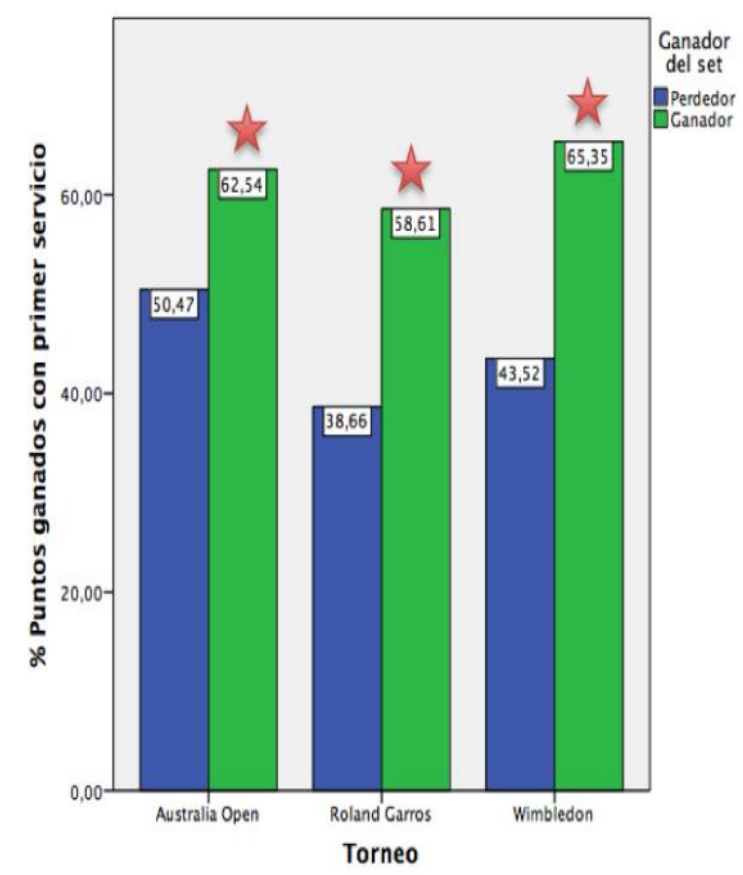

Figura 6. Descripción del \% puntos ganados con primer servicio en el TSR femenino. 


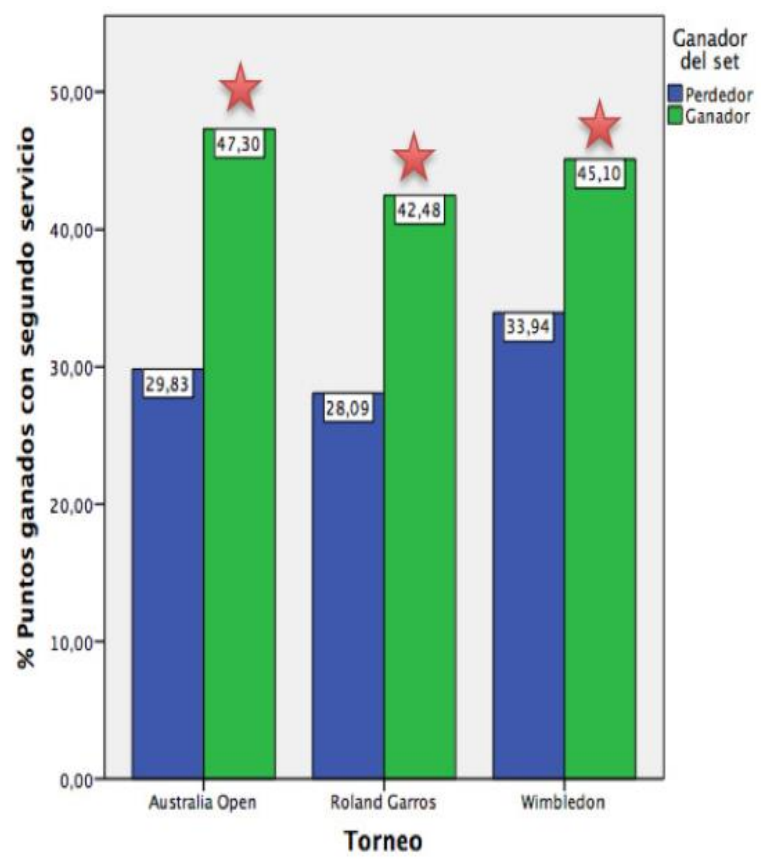

Figura 7. Descripción del \% puntos ganados con el segundo servicio en el TSR femenino.

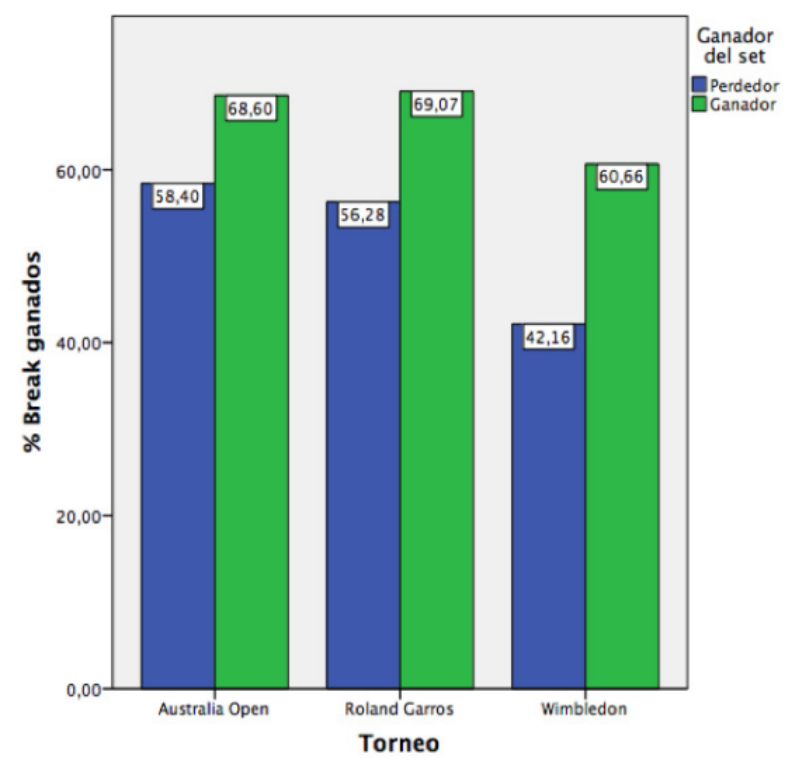

Figura 8. Descripción del \% rotura ganados del TSR femenino.

\section{DISCUSIÓN}

El análisis de los datos estadísticos de la competición aporta información de los requerimientos que tienen los jugadores en sus partidos, ayudando a mejorar la calidad de los entrenamientos con el objetivo de aumentar el rendimiento (Lago-Peñas, LagoBallesteros, Dellal, \& Gómez, 2010; Ortega,
Villarejo, \& Palao, 2009). El TSR puede ser jugado en diferentes superficies, y hasta la fecha no se tenían datos de partidos jugados en superficie de hierba, por lo que el presente trabajo se centrará en determinar las posibles diferencias respecto a la superficie de juego empleada (pista dura, pista de tierra batida y pista de hierba) en los torneos de Grand Slam, y analizar diferencias del rendimiento en el servicio de los jugadores ganadores y perdedores de élite.

El porcentaje de primer saque de los jugadores de TSR masculinos es ligeramente superior para el ganador del set que para el perdedor, aunque no se encuentran diferencias estadísticamente significativas (Figura 1). En cambio, las diferencias en el porcentaje de puntos ganados con primer saque (Figura 2) son superiores al $10 \%$ en todos los torneos $(p<.05)$. Esta diferencia de poco más del $10 \%$ es menor a los valores encontrados en la literatura entre ganadores y perdedores (47vs72\%) en pista dura (Sánchez-Pay, TorresLuque, Fernandez-García, Sanz-Rivas, \& Palao, 2017) en los Juegos Paralímpicos. Esto puede ser debido a la igualdad en los torneos de GS donde sólo compiten los ocho mejores del ranking internacional, por lo que la igualdad pueda ser mayor entre los jugadores. En relación al segundo servicio, los valores siguen la misma tendencia que con el primer servicio, excepto que en el torneo de Wimbledon la diferencia entre los ganadores y perdedores es mayor $(p<.05)$.

En relación al porcentaje break ganados, se observa que el torneo de RG no muestra diferencias entre el ganador y el perdedor del set; en cambio, los torneos de AO y W muestran porcentajes cercanos al $30 \%$ lo que pone de manifiesto que las superficies rápidas parecen afectar en mayor medida a las diferencias de nivel entre los dos jugadores. Esto puede entenderse como un indicador de igualdad en el resultado del partido, en el que se disputen mayor número de puntos por juego y se den mayor oportunidades de break en superficie lenta (RG) que en superficies rápidas ( $A O$ y UO) (Sánchez-Pay, Palao, et al., 2015). De igual manera, el hecho de que la velocidad del servicio no sea muy elevada, debido a la posición (plano de golpeo) del jugador y a la restricción para utilizar el tren inferior en la mecánica del movimiento (Cavedon, Zancanaro, \& Milanese, 2014; Reid, Elliott, \& Alderson, 2007), hace que el servicio sea más vulnerable que, por ejemplo en el tenis convencional, si además la superficie de la pista es más lenta, igualmente contribuye a que el servicio no sea tan comprometido para restarlo e iniciar el intercambio.

Por otra parte, debemos tener en cuenta que los jugadores en silla de ruedas, tras el servicio, tienen mayor dificultad de reaccionar y desplazarse rápidamente que los jugadores sin discapacidad, por lo que el resto se puede convertir en un golpe definitivo en muchos casos, sobre todo cuando la superficie contribuye en la velocidad de desplazamiento de la pelota tras el bote, aspecto que, nuevamente, resta protagonismo al servicio.

En relación a los datos en el TSR femenino, las diferencias entre las ganadoras y las perdedoras siguen la línea de lo comentado anteriormente para el género masculino, aunque con diferencias más marcadas para los porcentaje de puntos 
ganados con primer y segundo servicio así como en el porcentaje de break ganados en los tres torneos. Esto puede ser debido a que exista menor homogeneidad en el nivel de las participantes y haga que los partidos sean más desigualados.

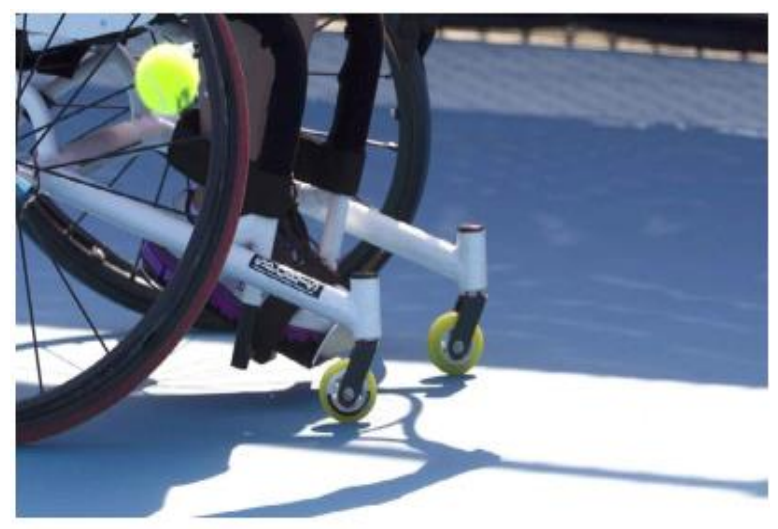

\section{CONCLUSIONES}

Con los resultados obtenidos en este trabajo relacionados con las estadísticas de competición en el tenis en silla de ruedas entre ganadores y perdedores en diferentes superficies de juego, se pueden establecer las siguientes conclusiones:

- El porcentaje de jugar con primer saque es similar entre los ganadores y perdedores tanto para el género masculino como para el femenino independientemente del torneo.

- El porcentaje de puntos ganados con el primer y segundo servicio en el TSR masculino es mayor para los ganadores del set que para los perdedores. En el TSR femenino las diferencias están más marcadas, por lo tanto, pese a que sea menos determinante en el tenis en silla, es muy importante intentar conseguir ventaja con el mismo, bien en términos de potencia y precisión, o en términos de desplazar al oponente mediante efectos.

- Las superficies rápidas ( $\mathrm{AO}$ y W) parecen afectar en mayor medida a las diferencias de nivel entre los ganadores y perdedores que la superficie lenta (RG).

\section{BIBLIOFRAFIA}

Cavedon, V., Zancanaro, C., \& Milanese, C. (2014). Kinematic analysis of the wheelchair tennis serve: Implications for classification. Scandinavian Journal of Medicine and Science in Sports, 24(5), 381-388. https://doi.org/10.1111/sms.12182

Cross, R., \& Pollard, G. (2009). Grand Slam men's singles tennis 19912009. Serve speeds and other related data. Coaching \& Sport Science Review, 16(49), 8-10.

ITF. (2018). Wheelchair Tennis Regulations. ITF. London.
Knight, G., \& O'Donoghue, P. (2012). The probability of winning break points in Grand Slam men's singles tennis. European Journal of Sport Science, 12(6), 462-468. https://doi.org/10.1080/17461391.2011.577239

Lago-Peñas, C., Lago-Ballesteros, J., Dellal, A., \& Gómez, M. (2010). Game-related statistics that discriminated winning, drawing and losing teams from the Spanish soccer league. Journal of Sports Science and Medicine, 9(2), 288-293.

Ortega, E., Villarejo, D., \& Palao, J. M. (2009). Differences in game statistics between winning and losing rugby teams in the six nations tournament. Journal of Sports Science and Medicine, $8(4), 523-527$.

Reid, M., Elliott, B., \& Alderson, J. (2007). Shoulder joint kinetics of the elite wheelchair tennis serve. British Journal of Sports Medicine, 41(11), 739-44. https://doi.org/10.1136/bjsm.2007.036145

Sánchez-Pay, A., Palao, J. M., Torres-Luque, G., \& Sanz-Rivas, D. (2015). Differences in set statistics between wheelchair and conventional tennis on different types of surfaces and by gender. International Journal of Performance Analysis in Sport, 15(3), 1177-1188. https://doi.org/10.1080/24748668.2015.11868860

Sánchez-Pay, A., Torres-Luque, G., Cabello Manrique, D., SanzRivas, D., \& Palao, J. M. (2015). Match analysis of women's wheelchair tennis matches for the Paralympic Games. International Journal of Performance Analysis in Sport, 15(1), 69-79. https://doi.org/10.1080/24748668.2015.11868777

Sánchez-Pay, A., Torres-Luque, G., Fernandez-García, Á. I., SanzRivas, D., \& Palao, J. M. (2017). Differences in game statistics between winning and losing for male wheelchair tennis players in Paralympics Games. Motriz: Revista de Educação Física, 23(3), 16. https://doi.org/10.1590/s1980-6574201700030011

\section{CONTENIDO ITF ACADEMY RECOMENDADO (HAZ CLICK ABAJO)}

\section{ITF Academy}

Derechos de Autor (c) 2018 Alejandro Sánchez, Antonio Ortega y David Sanz

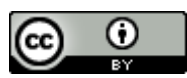

Este texto está protegido por una licencia CreativeCommons 4.0 .

Usted es libre para Compartir -copiar y redistribuir el material en cualquier medio o formato- y Adaptar el documento -remezclar, transformar y crear a partir del material- para cualquier propósito, incluso para fines comerciales, siempre que cumpla la condición de:

Atribución: Usted debe dar crédito a la obra original de manera adecuada, proporcionar un enlace a la licencia, e indicar si se han realizado cambios. Puede hacerlo en cualquier forma razonable, pero no de forma tal que sugiera que tiene el apoyo del licenciante o lo recibe por el uso que hace de la obra.

$\underline{\text { Resumendelicencia - Textocompletodelalicencia }}$ 